\title{
IMPULSIVE FUNCTIONAL-DIFFERENTIAL EQUATIONS WITH NONLOCAL CONDITIONS
}

\author{
HAYDAR AKÇA, ABDELKADER BOUCHERIF, and VALÉRY COVACHEV
}

Received 22 April 2001 and in revised form 7 August 2001

\begin{abstract}
The existence, uniqueness, and continuous dependence of a mild solution of an impulsive functional-differential evolution nonlocal Cauchy problem in general Banach spaces are studied. Methods of fixed point theorems, of a $C_{0}$ semigroup of operators and the Banach contraction theorem are applied.
\end{abstract}

2000 Mathematics Subject Classification: 34A37, 34G20, 34K30, 34K99.

1. Introduction. In this paper, we study the existence, uniqueness, and continuous dependence of a mild solution of a nonlocal Cauchy problem for impulsive functionaldifferential evolution equation. Such problems arise in some physical applications as a natural generalization of the classical initial value problems. The results for semilinear functional-differential evolution nonlocal problem [2] are extended for the case of impulse effect. We consider the nonlocal Cauchy problem in the form

$$
\begin{aligned}
\dot{u}(t) & =A u(t)+f\left(t, u_{t}\right), \quad t \in(0, a], t \neq \tau_{k}, \\
u\left(\tau_{k}+0\right) & =Q_{k} u\left(\tau_{k}\right) \equiv u\left(\tau_{k}\right)+I_{k} u\left(\tau_{k}\right), \quad k=1,2, \ldots, \kappa, \\
u(t) & +\left(g\left(u_{t_{1}}, \ldots, u_{t_{p}}\right)\right)(t)=\phi(t), \quad t \in[-r, 0],
\end{aligned}
$$

where $0<t_{1}<\cdots<t_{p} \leq a, p \in \mathbb{N}, A$ and $I_{k}(k=1,2, \ldots, \kappa)$ are linear operators acting in a Banach space $E ; f, g$, and $\phi$ are given functions satisfying some assumptions, $u_{t}(s):=u(t+s)$ for $t \in[0, a], s \in[-r, 0], I_{k} u\left(\tau_{k}\right)=u\left(\tau_{k}+0\right)-u\left(\tau_{k}-0\right)$ and the impulsive moments $\tau_{k}$ are such that $0<\tau_{1}<\tau_{2}<\cdots<\tau_{k}<\cdots<\tau_{\kappa}<a, \kappa \in \mathbb{N}$.

Theorems about the existence, uniqueness, and stability of solutions of differential and functional-differential abstract evolution Cauchy problems were studied in $[1,2,3]$. The results presented in this paper are a generalization and a continuation of some results reported in [1, 2, 3]. We consider classical impulsive functionaldifferential equation in the case of nonlocal condition, reduced to the classical initial functional value problem.

As usual, in the theory of impulsive differential equations $[4,5]$ at the points of discontinuity $\tau_{i}$ of the solution $t \mapsto u(t)$ we assume that $u\left(\tau_{i}\right) \equiv u\left(\tau_{i}-0\right)$. It is clear that, in general, the derivatives $\dot{u}\left(\tau_{i}\right)$ do not exist. On the other hand, according to the first equality of (1.1) there exist the limits $\dot{u}\left(\tau_{i} \mp 0\right)$. According to the above convention, we assume $\dot{u}\left(\tau_{i}\right) \equiv \dot{u}\left(\tau_{i}-0\right)$. 
Throughout, we assume that $E$ is a Banach space with norm $\|\cdot\|, A$ is the infinitesimal generator of a $C_{0}$ semigroup $\{T(t)\}_{t \geq 0}$ on $E, D(A)$ is the domain of $A$, and

$$
M:=\sup _{t \in[0, a]}\left\{\|T(t)\|_{B L(E, E)}\right\} .
$$

Let $f:[0, a] \times C([-r, 0], E) \rightarrow E$. Introduce the following assumptions:

(H1) for every $w \in C([-r, a], E)$ and $t \in[0, a], f\left(\cdot, w_{t}\right) \in C([0, a], E)$;

(H2) there exists a constant $L>0$ such that

$$
\begin{gathered}
\left\|f\left(t, w_{t}\right)-f\left(t, \tilde{w}_{t}\right)\right\|_{E} \\
\leq L_{1}\|w-\tilde{w}\|_{C([-r, t], E)} \quad \text { for } w, \tilde{w} \in C([-r, a], E), t \in[0, a], \\
\left\|I_{k} v\right\|_{E} \leq L_{2}\|v\|_{E} \quad \text { for } v \in E, k=1,2, \ldots, \kappa, \\
L=\max \left\{L_{1}, L_{2}\right\} .
\end{gathered}
$$

Let $g:[C([-r, 0], E)]^{p} \rightarrow C([-r, 0], E)$. Then we have the following assumptions:

(H3) there exists a constant $K>0$ such that

$$
\left\|\left(g\left(w_{t_{1}}, \ldots, w_{t_{p}}\right)\right)(t)-\left(g\left(\tilde{w}_{t_{1}}, \ldots, \tilde{w}_{t_{p}}\right)\right)(t)\right\| \leq K\|w-\tilde{w}\|_{C([-r, a], E)}
$$

for $w, \tilde{w} \in C([-r, a], E), t \in[-r, 0]$;

(H4) assume that $\phi \in C([-r, 0], E)$.

A function $u \in C([-r, a], E)$ satisfying the following conditions:

$$
\begin{aligned}
u(t)= & T(t) \phi(0)-T(t)\left[\left(g\left(u_{t_{1}}, \ldots, u_{t_{p}}\right)\right)(0)\right] \\
+ & \int_{0}^{t} T(t-s) f\left(s, u_{s}\right) d s+\sum_{0<\tau_{k}<t} T\left(t-\tau_{k}\right) I_{k} u\left(\tau_{k}\right), t \in[0, a], \\
& u(t)+\left(g\left(u_{t_{1}}, \ldots, u_{t_{p}}\right)\right)(t)=\phi(t), \quad t \in[-r, 0),
\end{aligned}
$$

is said to be a mild solution of the nonlocal Cauchy problem (1.1).

\section{Existence and uniqueness of a mild solution}

THEOREM 2.1. Suppose that assumptions (H1)-(H4) are satisfied and

$$
M[K+L(a+1)]<1 .
$$

Then the impulsive nonlocal Cauchy problem (1.1) has a unique mild solution.

Proof. The mild solution of the impulsive system (1.1) with nonlocal condition can be written in the form

$$
u(t ; \phi)=(F u)(t),
$$


where

$$
(F w)(t):=\left\{\begin{array}{l}
\phi(t)-\left(g\left(w_{t_{1}}, \ldots, w_{t_{p}}\right)\right)(t), \quad t \in[-r, 0), \\
T(t) \phi(0)-T(t)\left[\left(g\left(w_{t_{1}}, \ldots, w_{t_{p}}\right)\right)(0)\right] \\
\quad+\int_{0}^{t} T(t-s) f\left(s, w_{s}\right) d s+\sum_{0<\tau_{k}<t} T\left(t-\tau_{k}\right) I_{k} w\left(\tau_{k}\right), \quad t \in[0, a],
\end{array}\right.
$$

such that $w \in C([-r, a], E)$ and $F: C([-r, a], E) \rightarrow C([-r, a], E)$. Now, we show that $F$ is a contraction mapping on $C([-r, a], E)$. Therefore,

$$
(F w)(t)-(F \tilde{w})(t):=\left\{\begin{array}{c}
\left(g\left(\tilde{w}_{t_{1}}, \ldots, \tilde{w}_{t_{p}}\right)\right)(t)-\left(g\left(w_{t_{1}}, \ldots, w_{t_{p}}\right)\right)(t) \\
\text { for } w, \tilde{w} \in C([-r, a], E), t \in[-r, 0) \\
T(t)\left[\left(g\left(\tilde{w}_{t_{1}}, \ldots, \tilde{w}_{t_{p}}\right)\right)(0)-\left(g\left(w_{t_{1}}, \ldots, w_{t_{p}}\right)\right)(0)\right] \\
+\int_{0}^{t} T(t-s)\left[f\left(s, w_{s}\right)-f\left(s, \tilde{w}_{s}\right)\right] d s \\
+\sum_{0<\tau_{k}<t} T\left(t-\tau_{k}\right)\left[I_{k} w\left(\tau_{k}\right)-I_{k} \tilde{w}\left(\tau_{k}\right)\right] \\
\text { for } w, \tilde{w} \in C([-r, a], E), t \in[0, a] .
\end{array}\right.
$$

From (2.4), we have

$$
\begin{aligned}
\|(F w)(t)-(F \tilde{w})(t)\| \leq & \|T(t)\| \cdot\left\|\left(g\left(\tilde{w}_{t_{1}}, \ldots, \tilde{w}_{t_{p}}\right)\right)(0)-\left(g\left(w_{t_{1}}, \ldots, w_{t_{p}}\right)\right)(0)\right\| \\
& +\int_{0}^{t}\|T(t-s)\| \cdot\left\|f\left(s, w_{s}\right)-f\left(s, \tilde{w}_{s}\right)\right\| d s \\
& +\sum_{0<\tau_{k}<t}\left\|T\left(t-\tau_{k}\right)\right\| \cdot\left\|I_{k} w\left(\tau_{k}\right)-I_{k} \tilde{w}\left(\tau_{k}\right)\right\|
\end{aligned}
$$

for $w, \tilde{w} \in C([-r, a], E), t \in[0, a]$. Because of (2.5), in view of (1.2), and applying assumptions (H1)-(H4) we obtain

$$
\begin{aligned}
\|(F w)(t)-(F \tilde{w})(t)\| \leq & M K\|w-\tilde{w}\|_{C([-r, a], E)} \\
& +M L_{1} \int_{0}^{t}\|w-\tilde{w}\|_{C([-r, a], E)} d s+M L_{2}\left\|w\left(\tau_{k}\right)-\tilde{w}\left(\tau_{k}\right)\right\|_{E} \\
\leq & \left(M K+M a L_{1}+M L_{2}\right)\|w-\tilde{w}\|_{C([-r, a], E)} \\
\leq & M[K+L(a+1)] \cdot\|w-\tilde{w}\|_{C([-r, a], E)}
\end{aligned}
$$

for $w, \tilde{w} \in C([-r, a], E), t \in[0, a]$, which implies that

$$
\|F w-F \tilde{w}\|_{C([-r, a], E)} \leq \beta\|w-\tilde{w}\|_{C([-r, a], E)}, \quad w, \tilde{w} \in C([-r, a], E),
$$

where $\beta:=M[K+L(a+1)]$. The operator $F$ satisfies all the assumptions of the Banach contraction theorem, and therefore, in the space $C([-r, a], E)$ there is only one fixed point of $F$ and this is the mild solution of the nonlocal Cauchy problem with impulse effect. This completes the proof of the theorem. 


\section{Continuous dependence of a mild solution}

THEOREM 3.1. Suppose that the functions $f, g$, and $I_{k}(u), k=1,2, \ldots, \kappa$, satisfy the assumptions (H1)-(H4) and $M[K+L(a+1)]<1$. Then, for each $\phi_{1}, \phi_{2} \in C([-r, a], E)$, and for the corresponding mild solutions $u_{1}, u_{2}$ of the problems,

$$
\begin{gathered}
\dot{u}(t)=A u(t)+f\left(t, u_{t}\right), \quad t \in(0, a], t \neq \tau_{k}, \\
u\left(\tau_{k}+0\right)=Q_{k} u\left(\tau_{k}\right) \equiv u\left(\tau_{k}\right)+I_{k} u\left(\tau_{k}\right), \quad k=1,2, \ldots, \kappa, \\
u(t)+\left(g\left(u_{t_{1}}, \ldots, u_{t_{p}}\right)\right)(t)=\phi_{i}(t) \quad(i=1,2), t \in[-r, 0],
\end{gathered}
$$

the following inequality holds:

$$
\left\|u_{1}-u_{2}\right\|_{C([-r, a], E)} \leq M e^{a M L}(1+M L)^{\kappa}\left\{\left\|\phi_{1}-\phi_{2}\right\|_{C([-r, 0], E)}+K\left\|u_{1}-u_{2}\right\|_{C([-r, a], E)}\right\} .
$$

Additionally, if

$$
K<\frac{e^{-a M L}(1+M L)^{-\kappa}}{M}
$$

then

$$
\left\|u_{1}-u_{2}\right\|_{C([-r, a], E)} \leq \frac{M e^{a M L}(1+M L)^{\kappa}}{1-K M e^{a M L}(1+M L)^{\kappa}}\left\|\phi_{1}-\phi_{2}\right\|_{C([-r, 0], E)} .
$$

Proof. Assume that $\phi_{i} \in C([-r, 0], E)(i=1,2)$ are arbitrary functions and let $u_{i}$ $(i=1,2)$ be the mild solutions of problem (3.1). Then

$$
\begin{aligned}
u_{1}(t)-u_{2}(t)= & T(t)\left[\phi_{1}(0)-\phi_{2}(0)\right] \\
& -T(t)\left\{\left[g\left(\left(u_{1}\right)_{t_{1}}, \ldots,\left(u_{1}\right)_{t_{p}}\right)\right](0)-\left[g\left(\left(u_{2}\right)_{t_{1}}, \ldots,\left(u_{2}\right)_{t_{p}}\right)\right](0)\right\} \\
& +\int_{0}^{t} T(t-s)\left[f\left(s,\left(u_{1}\right)_{s}\right)-f\left(s,\left(u_{2}\right)_{s}\right)\right] d s \\
& +\sum_{0<\tau_{k}<t} T\left(t-\tau_{k}\right)\left[I_{k} u_{1}\left(\tau_{k}\right)-I_{k} u_{2}\left(\tau_{k}\right)\right]
\end{aligned}
$$

for $t \in[0, a]$ and

$$
u_{1}(t)-u_{2}(t)=\phi_{1}(t)-\phi_{2}(t)-\left\{\left[g\left(\left(u_{2}\right)_{t_{1}}, \ldots,\left(u_{2}\right)_{t_{p}}\right)\right](t)-\left[g\left(\left(u_{1}\right)_{t_{1}}, \ldots,\left(u_{1}\right)_{t_{p}}\right)\right](t)\right\}
$$

for $t \in[-r, 0)$. From (3.5), (1.2), and using (H2) we get

$$
\begin{aligned}
\left\|u_{1}(\xi)-u_{2}(\xi)\right\| \leq & M\left\|\phi_{1}-\phi_{2}\right\|_{C([-r, 0], E)}+M K\left\|u_{1}-u_{2}\right\|_{C([-r, a], E)} \\
& +M L_{1} \int_{0}^{\xi}\left\|u_{1}-u_{2}\right\|_{C([-r, s], E)} d s+M L_{2} \sum_{0<\tau_{k}<\xi}\left\|u_{1}\left(\tau_{k}\right)-u_{2}\left(\tau_{k}\right)\right\|_{E} \\
\leq & M\left\|\phi_{1}-\phi_{2}\right\|_{C([-r, 0], E)}+M K\left\|u_{1}-u_{2}\right\|_{C([-r, a], E)} \\
& +M L_{1} \int_{0}^{t}\left\|u_{1}-u_{2}\right\|_{C([-r, s], E)} d s+M L_{2} \sum_{0<\tau_{k}<t}\left\|u_{1}\left(\tau_{k}\right)-u_{2}\left(\tau_{k}\right)\right\|_{E}
\end{aligned}
$$


for $0 \leq \xi \leq t \leq a$. With this result, by virtue of (H3) it follows that

$$
\begin{aligned}
\sup _{\xi \in[0, t]} & \left\|u_{1}(\xi)-u_{2}(\xi)\right\| \\
\leq & M\left\|\phi_{1}-\phi_{2}\right\|_{C([-r, 0], E)}+M K\left\|u_{1}-u_{2}\right\|_{C([-r, a], E)} \\
& +M L_{1} \int_{0}^{t}\left\|u_{1}-u_{2}\right\|_{C([-r, s], E)} d s+M L_{2} \sum_{0<\tau_{k}<t}\left\|u_{1}\left(\tau_{k}\right)-u_{2}\left(\tau_{k}\right)\right\|_{E}
\end{aligned}
$$

for $t \in[0, a]$. At the same time, by (3.6) and (H3) we have

$$
\left\|u_{1}(t)-u_{2}(t)\right\| \leq M\left\|\phi_{1}-\phi_{2}\right\|_{C([-r, 0], E)}+M K\left\|u_{1}-u_{2}\right\|_{C([-r, a], E)}
$$

for $t \in[-r, 0)$. Formulas (3.8) and (3.9) imply that

$$
\begin{aligned}
\left\|u_{1}(t)-u_{2}(t)\right\| \leq & M\left\|\phi_{1}-\phi_{2}\right\|_{C([-r, 0], E)}+M K\left\|u_{1}-u_{2}\right\|_{C([-r, a], E)} \\
& +M L\left\{\int_{0}^{t}\left\|u_{1}-u_{2}\right\|_{C([-r, s], E)} d s+\sum_{0<\tau_{k}<t}\left\|u_{1}\left(\tau_{k}\right)-u_{2}\left(\tau_{k}\right)\right\|_{E}\right\} .
\end{aligned}
$$

Applying Gronwall's inequality for discontinuous functions (see [5]), from (3.10) it follows that

$$
\begin{aligned}
\left\|u_{1}(t)-u_{2}(t)\right\|_{C([-r, a], E)} \leq & \left\{M\left\|\phi_{1}-\phi_{2}\right\|_{C([-r, 0], E)}\right. \\
& \left.+M K\left\|u_{1}-u_{2}\right\|_{C([-r, a], E)}\right\} e^{a M L}(1+M L)^{\kappa}
\end{aligned}
$$

and therefore, (3.2) holds. Inequality (3.4) is a consequence of (3.2). This completes the proof of the theorem.

REMARK 3.2. If $K=\kappa=0$, then (3.2) is reduced to the classical inequality

$$
\left\|u_{1}(t)-u_{2}(t)\right\|_{C([-r, a], E)} \leq M e^{a M L}\left\|\phi_{1}-\phi_{2}\right\|_{C([-r, 0], E)},
$$

which is characteristic for the continuous dependence of the semilinear functionaldifferential evolution Cauchy problem with the classical initial condition.

ACKNOWLEDGEMENT. The authors would like to thank King Fahd University of Petroleum and Minerals, Department of Mathematical Sciences for providing excellent research facilities. The present research was accomplished during the stay of the third author at Fatih University, Istanbul, Turkey.

\section{REFERENCES}

[1] H. Akça and V. Covachev, Periodic solutions of impulsive systems with delay, Funct. Differ. Equ. 5 (1998), no. 3-4, 275-286.

[2] L. Byszewski and H. Akça, On a mild solution of a semilinear functional-differential evolution nonlocal problem, J. Appl. Math. Stochastic Anal. 10 (1997), no. 3, 265-271.

[3] __ Existence of solutions of a semilinear functional-differential evolution nonlocal problem, Nonlinear Anal. 34 (1998), no. 1, 65-72.

[4] V. Lakshmikantham, D. D. Baĭnov, and P. S. Simeonov, Theory of Impulsive Differential Equations, Modern Applied Mathematics, vol. 6, World Scientific Publishing, New Jersey, 1989. 
[5] A. M. Samoĭlenko and N. A. Perestyuk, Impulsive Differential Equations, World Scientific Series on Nonlinear Science. Series A: Monographs and Treatises, vol. 14, World Scientific Publishing, New Jersey, 1995.

Haydar akça: Department of Mathematical Sciences, King Fahd University of Petroleum AND Minerals, DHAHran 31261, SAUdi ARABiA

E-mail address: akca@kfupm. edu.sa

ABDELKADER BOUCHERIF: DePARTMENT OF MATHEMATICAL SCIENCES, King FAHD UNIVERSITY of Petroleum and Minerals, Dhahran 31261, Saudi Arabia

E-mail address: aboucher@kfupm.edu.sa

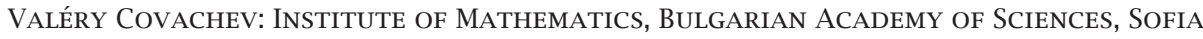
1113, BULGARIA

E-mail address: matph@math. bas.bg 


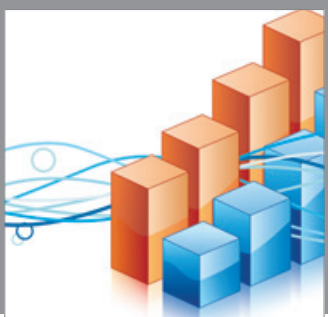

Advances in

Operations Research

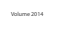

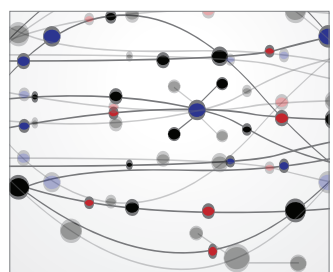

\section{The Scientific} World Journal
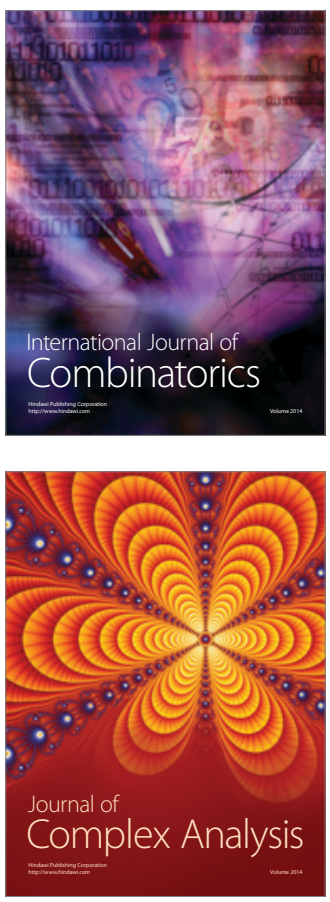

International Journal of

Mathematics and

Mathematical

Sciences
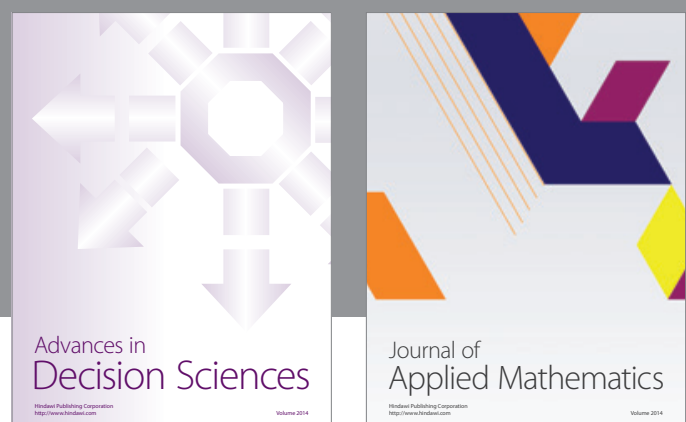

Journal of

Applied Mathematics
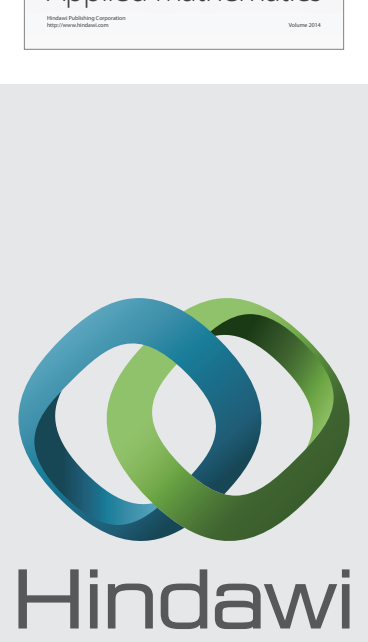

Submit your manuscripts at http://www.hindawi.com
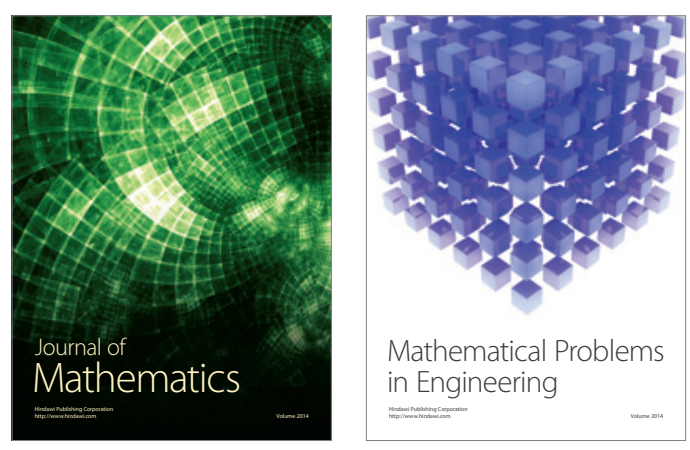

Mathematical Problems in Engineering
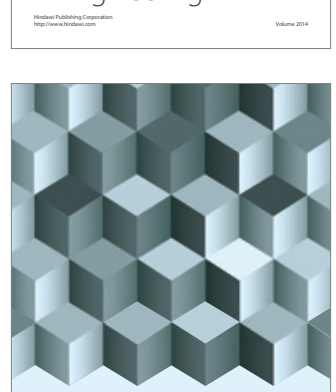

Journal of

Function Spaces
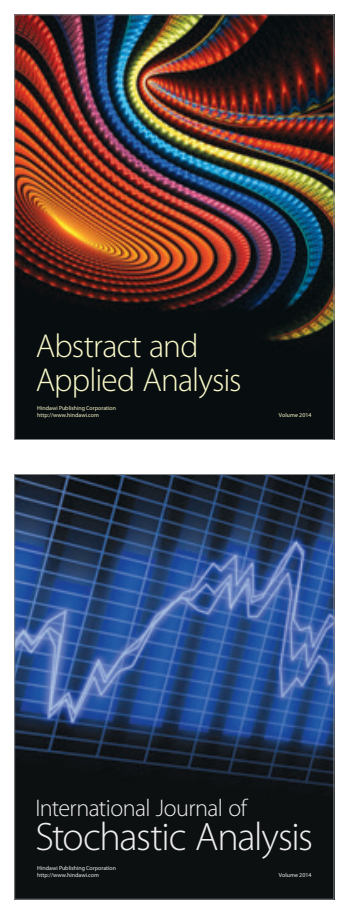

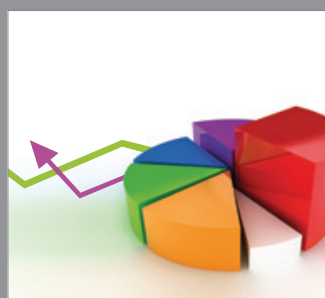

ournal of

Probability and Statistics

Promensencen
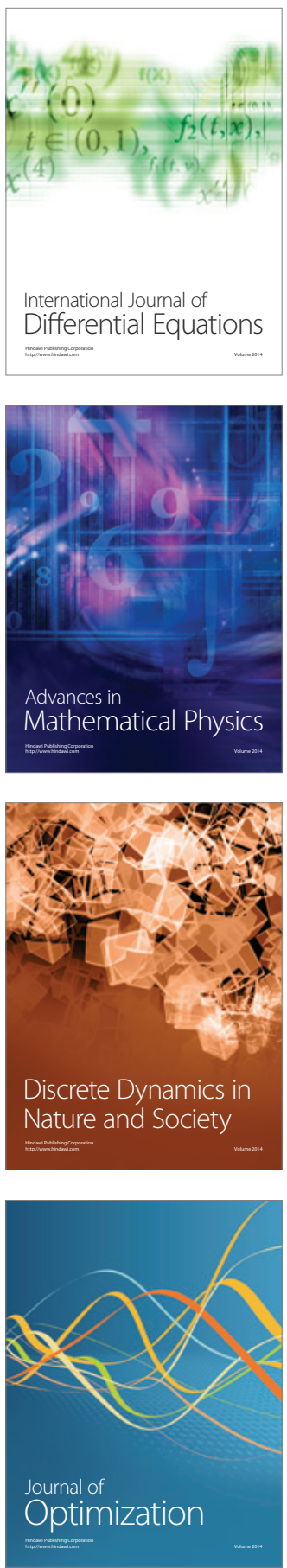